\title{
Interactive comment on "Shallow water table effects on water, sediment and pesticide transport in vegetative filter strips: Part B. model coupling, application, factor importance and uncertainty" by Claire Lauvernet and Rafael Muñoz-Carpena
}

\section{Anonymous Referee \#3}

Received and published: 2 October 2017

General Comments: This manuscript demonstrates the integration of a new infiltration model that accounts for a shallow water table with the VFSMOD vegetative filter strop simulation model. The paper's title and abstract to an excellent job of reflecting the emphasis and content of the paper, and overall, the manuscript is clearly structured and reads very well. The modeling analysis in this paper compares the version of VFSMOD that does not include a shallow water table with the new version that does include the shallow water table and new infiltration model. The novelty of this paper is with regard to the application of the new infiltration model that accounts for the shallow water table, 
and the impacts that accounting for this has on VFS effectiveness if reducing sediment, runoff, and pollutants. The analysis of the new modeling approach includes application of the models to 2 field sites for comparison with observed VFS behavior, and a global sensitivity analysis and uncertainty analysis to identify parameters responsible for the efficiency of VFSs with and without shallow water table conditions. The conclusions highlight the significant influence that a shallow water table has on VFS effectiveness, as well as the additional complexity that the presence of a shallow water has on simulation of VFSs. The authors provide a good level of discussion providing necessary background and references concerning VFS simulation, the novel infiltration simulation model, study site data, and the GSA methods used to assess the model sensitivity and uncertainty As with Part A of this manuscript, the methods and assumption are clearly described, and the results sufficiently support many of the conclusions; however, the validation of the model with observed field data is limited.

Specific Comments: 1. Visual comparison of the simulated versus modeled VFS outflow is provided in Figure 4a for 1 event. Could the authors provide some goodness of fit statistics, such as Nash-Sutcliffe efficiency or others in addition to the visual interpretation? 2. Why are observations at the Jailliere not shown for comparison with the model simulation in Figure $4 b$ ? Even if detailed time series are not available, an event total volume could be compared between model and observations. 3 . The description of the study sites indicates that monitoring was conducted over a multi-year period at each site, and that around 20 events per site were analyzed. However, observations from only 1 event at 1 site were included for review and discussion in the manuscript. This leaves the reader wondering how the model performs for all the other storm events. It is important for readers to see how the model performs over a broader range of events, as 1 single event at one site is not enough to assess the validity of the model. The authors should provide the model simulation comparisons with observed data for the complete set of events available at each site which is necessary to better validate the new approach and any improvement it has over the approach that does not include the shallow water table. 4 . The model performance compared to observa-

Printer-friendly version

Discussion paper 
tions at the Morcille site was fair, and the authors deemed the model performance as satisfactory given that the model was not calibrated. If the authors were to provide an example of the performance of the model when calibrated, readers would better understand the potential improvement in a calibrated model and be better able to make a judgement regarding the validity of this new modeling approach. 5 . Figure $4 \mathrm{a}, 4 \mathrm{~b}$ : In both figures, the legends are incomplete. Symbology for all time series shown in the figures should be included. 6. Figure 5: The marker symbols that are included as part of several of the lines should be larger. The line symbology should be more distinct for the dashed lines if possible. 7. Figure 6: The labeling for the majority of the points on these plots in not legible. The labels that cannot be read should be removed from each plot. Labels should only be included for points of greatest significance or ones that are referred to in the text, and must be legible if included. 8. Figure 7: The x-axis labels are pretty tough to read because there are so many parameters shown. This could be potentially fixed by not showing some of the parameter results in the plot. 9. Figure 8: The symbology is a little difficult to match between the legend and the figures. Part of the reason for this is that "dE" is concentrated up against the $100 \%$ level of the $x$-axis (accept for Figure 8b). One solution to improve this would be to also label each of the curves with an "callout" or arrow.

Interactive comment on Hydrol. Earth Syst. Sci. Discuss., https://doi.org/10.5194/hess-2017406, 2017. 